\title{
Magnetic Nanostructures for Lateral Spin-Transport Devices
}

\author{
Dirk Grundler, Thomas M. Hengstmann, and Haiko Rolff \\ Institut für Angewandte Physik und Zentrum für Mikrostrukturforschung, Universität Hamburg \\ Jungiusstrasse 11, D-20355 Hamburg, Germany
}

Received on 31 March, 2003

\begin{abstract}
We review our investigations on nanostructured ferromagnets fabricated on semiconductor heterostructures. We show that ballistic electrons in the semiconductor are in particular sensitive to local stray fields. The reversal of the ferromagnets give rise to characteristic magnetoresistance traces in the ferromagnet/semiconductor hybrid structures. We use these stray-field effects for detailed studies on the magnetic properties of rectangular micromagnets, nanodisks and nanorings. The nanostructures might be favorable candidates for ferromagnetic contacts in future spin-injection experiments in lateral feromagnet/semiconductor hybrid structures.
\end{abstract}

\section{Introduction}

Injection of spin-polarized currents into semiconductors is a challenging research field [1-4]. High spin injection efficiencies would render spintronics with semiconductors relevant for technological applications [5]. One of the most controversial issues in experiment [6-9] and theory [10] is the all-electrical spin injection and spin detection in a highmobility two-dimensional electron system (2DES) using ferromagnetic metals. We have recently developed a model to describe ballistic transport in ferromagnet/semiconductor hybrid structures [11-13]. In this transport regime spin injection is possible by means of spin filtering at the ferromagnet/semiconductor interface $[12,14]$. Characteristic and pronounced spin-valve effects are predicted [13]. It has earlier been argued however that stray fields perpendicular to the plane of the 2DES could induce an additional magnetoresistance (MR) effect which might mimic a spin-valve effect [8]. This Lorentz-force induced MR effect is often called the local Hall effect. The latter is prominent in lateral ferromagnet/semiconductor hybrid structures rather than in the all-metal giant magnetoresistance (GMR) devices due to the smaller carrier density $n_{s}$ of the 2DES. An important prerequisite for the interpretation of transport phenomena in ferromagnet/semiconductor hybrid structures is therefore a good knowledge of the stray fields of the ferromagnetic contacts. We have investigated different nanostructured ferromagnets with respect to the local Hall effect. Here, we review our recent experimental results.

\section{Experimental setup}

Mesoscopic 2DES which are sensitive to the local Hall effect $[15,16]$ have been fabricated in a $\mathrm{GaAs} / \mathrm{Al}_{0.3} \mathrm{Ga}_{0.7} \mathrm{As}$ heterostructure using electron-beam lithography and photolithography followed by a wet etching process. The heterostructure has been grown by means of molecular beam epitaxy (MBE). Due to modulation doping it contains a high-mobility 2DES $90 \mathrm{~nm}$ below the surface. The electronic width of the etched Hall bar is in the order of $1 \mu \mathrm{m}$. A typical device is shown in Fig. 1. Contacts are fabricated using evaporation and lift-off technique. The carrier density $n_{s}$ is $2.7 \cdot 10^{11} \mathrm{~cm}^{-2}$ and the mobility $\mu$ is $350,000 \mathrm{~cm}^{2} / \mathrm{Vs}$ at $4.2 \mathrm{~K}$. Hence, the electron mean free path is $l_{e}=3 \mu \mathrm{m}$. All experiments are performed at $4.2 \mathrm{~K}$ such that the electron system is in the ballistic transport regime. The polycrystalline permalloy $\left(\mathrm{Ni}_{80} \mathrm{Fe}_{20}\right)$ micro- and nanomagnets are integrated on the cap layer of the heterostructure using electron beam lithography, evaporation and lift-off technique (Fig. 1). The $20 \mathrm{~nm}$ thick permalloy film has been capped by a $9 \mathrm{~nm}$ gold layer. The ferromagnetic nanostructures are electrically isolated from the 2DES. By this means, we are able to investigate in detail the stray field effects and to focus on Lorentz-force induced MR effects. For this, we have performed Hall and bend-resistance measurements on the mesoscopic 2DES. The bend resistance is a characteristic MR feature that originates from electrons travelling ballistically through the 2DES [17].

The external magnetic field $B$ is applied in the plane of the 2DES, i.e., the observed hysteretic features are solely due to stray fields of the ferromagnets which are perpendicular to the 2DES. The field $B$ is swept in small steps of a few tenth of a mT. The current $I$ of amplitude $3 \mu \mathrm{A}$ applied to the 2DES is modulated at $37 \mathrm{~Hz}$. The voltage is detected using lock-in technique.

\section{Experimental results and discus- sion}

In the following we present stray-field investigations performed on three 2DES with different ferromagnetic nanostructures on top: (I) a rectangular two-micromagnet system, 


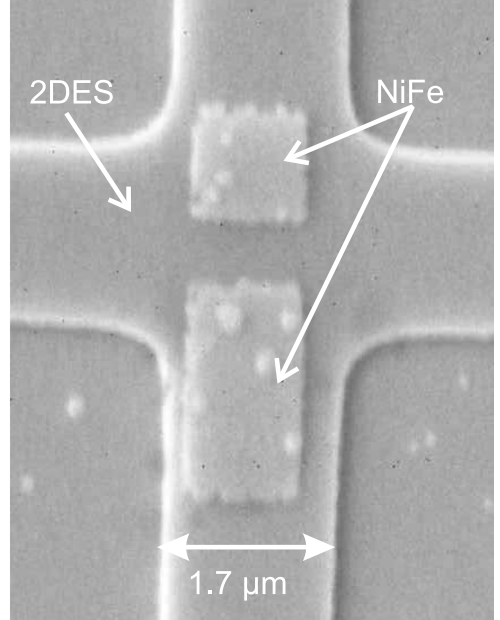

Figure 1. A two-micromagnet system fabricated from permalloy on top of the insulating cap layer of a Hall bar etched in a 2DES.

(II) a nanodisk and (III) a nanoring. The first has been proposed for spin-injection and spin-detection experiments in lateral ferromagnet/semiconductor hybrid structures in Ref. [18]. Nanodisks and nanorings have been proposed earlier to be interesting for GMR spin-valve devices [19].

\section{Rectangular micromagnets}

The Hall resistance measures the averaged magnetic flux in the 2DES $[15,16]$. Recently we have shown that, in particular, the bend resistance is sensitive to the local details of the field pattern penetrating the 2DES and that it is useful to enhance considerably the lateral resolution of a 2DES Hall bar [20]. This is demonstrated on sample (I) in Fig. 2. Here, the bend-resistance trace is shown which is measured on a 2DES cross junction containing a two-micromagnet system (compare Fig. 1) with a separation $d=200 \mathrm{~nm}$. The four contact leads are labelled with 1 to 4 (inset of Fig. 2). The current is injected between the leads 2 and 4 and the voltage is measured between the contacts 1 and 3. By this means, electrons are detected at contact 1 which transverse the cross junction ballistically [17] and which enter the voltage probe 1 without being deflected by the inhomogeneous stray field.

The bend resistance $R_{24,13}$ shows a hysteretic 'butterfly'-like shape which is interesting by itself. Here it is important that the stray field gives rise to a characteristic and pronounced MR effect for the ballistic electrons in the mesoscopic 2DES. In micromagnetic simulations we have found that the change of the domain pattern in the twomicromagnet system occurs very local within sub-micron domains. The experimental result is that ballistic electrons in the 2DES are very sensitive to these local stray fields [20], i.e., the Lorentz-force induced MR effect reflects in detail the magnetic behavior of the ferromagnets on the nanometer scale. In Fig. 2 the bend resistance is nonzero for almost all magnetic fields. Near zero magnetic field, i.e., in the remanent state, the bend resistance is in particular either positive or negative depending on the magnetic history. As a result, we find that a ballistic 2DES beneath a rectangular two-micromagnet system shows a very complex MR caused by the stray fields.

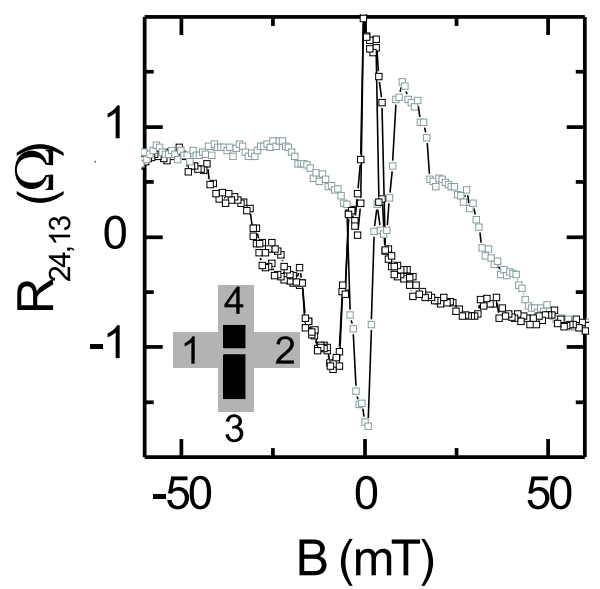

Figure 2. Bend resistance measured on a $2 \mathrm{DES}$ cross junction beneath a two-micromagnet system. The current is injected through the leads 2 and 4, the voltage drop is measured between the leads 1 and 3 . The configuration and the lead numbers are sketched in the inset. The zero-field resistance value has been subtracted from the data. Data were taken from Ref. [21].

\section{Nanodisks}

It has early been argued [19] that nanomagnets with a circular shape fabricated from permalloy might be a favorable candidate for spin-valve devices. Only very recently the intriguing magnetic properties of nanodisks have been verified experimentally [22]. In the remanent state of a nanodisk the microscopic magnetic moments should be in the plane of the disk and arranged in a clockwise or counterclockwise manner. In the center, there should exist a vortex which would give rise to a small stray field perpendicular to the plane of the disk.

We have fabricated nanodisks on mesoscopic 2DES and have investigated the stray-field effects [23]. The MR effect is found to be less complex if compared to rectangular micromagnets (Fig. 3). The important finding is that the local Hall effect follows very nicely the calculated hysteretic magnetization loop.

A comparison between simulated magnetization $M$ and Hall measurement $R_{H}$ is shown in Fig. 3 [sample(II)]. We find a good qualitative agreement. Only at zero magnetic field, i.e., in the remanent state, we observe a small discrepancy between the local Hall effect and the magnetization curve $M$ vs. $B$. In the experimental data, there is an offset in $R_{H}$ between the up sweep and the down sweep of the magnetic field $B$. This seems to reflect the chirality of the remanent magnetic state of the nanodisk and the orientation of the vortex which is formed in the central region of the nanodisk.

\section{Nanorings}

The small offset in $R_{H}$ in the remanent state, which is found in case of the nanodisk depending on the magnetic 


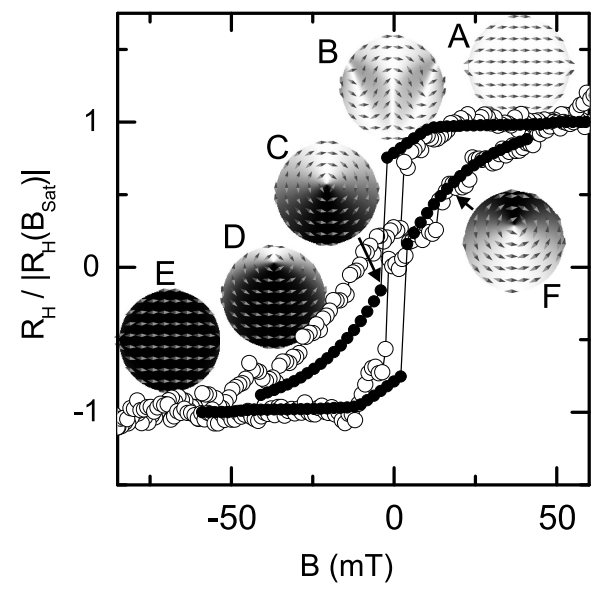

Figure 3. Comparison between the data of the Hall resistance $R_{H}$ of a 2DES beneath a permalloy nanodisk with $d=700 \mathrm{~nm}$ and thickness $t=20 \mathrm{~nm}$ (open symbols) and a simulated magnetization curve $M$ vs. $B$ (filled symbols). Both data show large irreversible jumps near zero field. Calculated domain configurations are depicted in a greyscale plot for the variation of $B$ from positive to negative values (A-E) and one configuration for the reverse sweep (F), the arrows indicate the direction of the magnetization vectors. Graph and data are taken from Ref. [23].

history, is removed in case of sample (III). In Fig. 4 we show the Hall effect in a mesoscopic 2DES which was beneath a permalloy nanoring. Here, mainly two magnetic configurations are distinguished: firstly, at high positive field the nanoring is in the so-called onion state [24] where both arms are magnetized in the same direction along the applied field. Secondly, close to zero field the ring is expected to be in a circular state, i.e., the microscopic magnetic moments are aligned in a ring-shaped fashion. If we reverse the magnetic field before the irreversible jump to the opposite onion state occurs, we find that the measured Hall voltage in the remanent state remains in the center of the hysteretic trace.

In the scenario of the ring-shaped magnetization state, the stray field of the nanoring vanishes in zero magnetic field since, if compared to the nanodisk, the perpendicular vortex is no longer present.

\section{Conclusion}

We have shown that the magnetic behavior of permalloy nanostructures is governed by the shape. In case of ringshaped nanomagnets the stray-field effects in the remanent state can be vanishingly small. Our findings might be helpful for the design of ferromagnetic contacts in lateral ferromagnet/semiconductor spin-valve devices in future spininjection experiments.

\section{Acknowledgements}

D. Grundler thanks E. A. de Andrada e Silva for his very kind hospitality during the stay at INPE in Sao Jose dos Campos and FAPESP and DAAD for the financial support. The work has been supported by the DFG via He1938/9 and via the Graduiertenkolleg 'Nanostrukturierte Festkörper'.

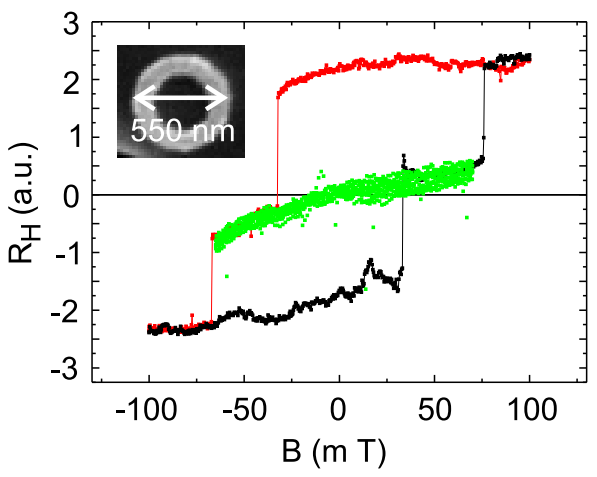

Figure 4. Local Hall effect measured in a mesoscopic 2DES at $4.2 \mathrm{~K}$ which was beneath a permalloy nanoring with an outer diameter of $550 \mathrm{~nm}$ and an inner diameter of $300 \mathrm{~nm}$. An offset is subtracted from the curves such that the absolute Hall voltage at maximum positive and minimum negative $B$ is the same. The inner data reflect several up and down sweeps where the field $B$ is always between the switching fields to the saturated onion states. Data taken from Ref. [25].

We gratefully acknowledge continuous support of our work by D. Heitmann, experimental help by N. Klockmann and MBE growth of the semiconductor heterostructures by $\mathrm{Ch}$. Heyn.

\section{References}

[1] S. Datta and B. Das, Appl. Phys. Lett. 56, 665 (1990)

[2] D.D. Awschalom, M.E. Flatte and N. Samarth, Sci. Am. 286, 66 (2002)

[3] D. Grundler, Phys. World 15 (no. 4), 39 (2002)

[4] G. Meier and D. Grundler, in Festkörperprobleme - Advances in Solid State Physics/Vol. 40, Ed. B. Kramer (Braunschweig: Vieweg 2000) p 295; and references therein

[5] S.A. Wolf et al., Science 294, 1488 (2001); and references therein

[6] P. Hammar, B.R. Bennett, M.J. Yang, and M. Johnson, Phys. Rev. Lett. 83, 203 (1999); Phys. Rev. Lett. 84, 5024 (2000)

[7] A.T. Filip, B. H. Hoving, F. J. Jedema, B.J. van Wees, B. Dutta, and S. Borghs, Phys. Rev. B 62, 9996 (2000)

[8] F.G. Monzon, H.X. Tang, and M.L. Roukes, Phys. Rev. Lett. 84, $5022(2000)$

[9] B.J. van Wees, Phys. Rev. Lett. 84, 5023 (2000)

[10] J.-I. Inoue, G.E. Bauer, and L.W. Molenkamp, Phys. Rev. B 67, 033104 (2003); and references therein

[11] D. Grundler, Phys. Rev. Lett. 86, 1058 (2001)

[12] D. Grundler, Phys. Rev. B 63, R161307 (2001)

[13] T. Matsuyama, C.-M. Hu, D. Grundler, G. Meier, and U. Merkt, Phys. Rev. B 65, 155322 (2002)

[14] O. Wunnicke, Ph. Mavropoulos, R. Zeller, P.H. Dederichs, and D. Grundler, Phys. Rev. B 65, 241306(R) 2002.

[15] A. Geim, S. V. Dubonos, J. G. S. Lok, I. V. Grigorieva, J. C. Maan, L. Theil Hansen, and P. E. Lindelof, Appl. Phys. Lett. 71, 2379 (1997). 
[16] G. Meier, D.Grundler, K.-B. Broocks, Ch. Heyn, and D. Heitmann, JMMM 210, 138 (2000).

[17] Y. Takagaki et. al., Solid State Comm. 68, 1051 (1988).

[18] G. Meier, M. Halverscheid, T. Matsuyama, and U. Merkt, J. Appl. Phys. 89, 7469 (2001).

[19] G. Prinz, US patent 5,542,868 and US patent $6,381,170$.

[20] D. Grundler, T. M. Hengstmann, N. Klockmann, Ch. Heyn, and D. Heitmann, Physica E 12, 248 (2002).
[21] T. M. Hengstmann, D. Grundler, N. Klockmann, H. Rolff, Ch. Heyn, and D. Heitmann, IEEE Trans. Magn. 38, 2535 (2002).

[22] R.P. Cowburn et al., Phys. Rev. Lett. 83,1042 (1999).

[23] T.M. Hengstmann, D. Grundler, Ch. Heyn, and D. Heitmann, J. Appl. Phys. 90, 6542 (2001).

[24] M. Kläui et al., Appl. Phys. Lett. 78, 3268 (2001).

[25] H. Rolff, Ph D thesis, University of Hamburg, 2003. 